\title{
Comparison of the Short-Term Forecasting Accuracy on Battery Electric Vehicle between Modified Bass and Lotka-Volterra Model: A Case Study of China
}

\author{
Shunxi Li, Hang Chen, and Guofang Zhang \\ School of Automotive Engineering, Hubei Key Laboratory of Advanced Technology for Automotive Components and Hubei \\ Collaborative Innovation Center for Automotive Components Technology, Wuhan University of Technology, Hubei 430070, China
}

Correspondence should be addressed to Shunxi Li; 1sx@whut.edu.cn

Received 7 April 2017; Accepted 4 July 2017; Published 7 August 2017

Academic Editor: Xing Wu

Copyright (C) 2017 Shunxi Li et al. This is an open access article distributed under the Creative Commons Attribution License, which permits unrestricted use, distribution, and reproduction in any medium, provided the original work is properly cited.

\begin{abstract}
The potential demand of battery electric vehicle (BEV) is the base of the decision-making to the government policy formulation, enterprise manufacture capacity expansion, and charging infrastructure construction. How to predict the future amount of BEV accurately is very important to the development of BEV both in practice and in theory. The present paper tries to compare the short-term accuracy of a proposed modified Bass model and Lotka-Volterra (LV) model, by taking China's BEV development as the case study. Using the statistics data of China's BEV amount of 21 months from Jan 2015 to Sep 2016, we compare the simulation accuracy based on the value of mean absolute percentage error (MAPE) and discuss the forecasting capacity of the two models according to China's government expectation. According to the MAPE value, the two models have good prediction accuracy, but the Bass model is more accurate than LV model. Bass model has only one dimension and focuses on the diffusion trend, while LV model has two dimensions and mainly describes the relationship and competing process between the two populations. In future research, the forecasting advantages of Bass model and LV model should be combined to get more accurate predicting effect.
\end{abstract}

\section{Introduction}

To resolve the problem of environmental pollution and energy shortage, the development of new energy vehicles has been paid much attention. China has become one of the fastest growing markets in the world. How to predict the future amount of BEV accurately is very important to the development of BEV both in practice and in theory.

The time series model and the causal prediction method have been done on the forecasting of car ownership in the following research. Based on the GDP growth rate, Yini (2005) used the Gompertz model to analysis the future vehicle ownership in China [1]. Gu et al. (2010) made a forecast on the vehicle ownership based on the provincial data of China [2]. Ma et al. (2009) used the AHP method and logit regression model to forecast the market share of new energy vehicles in China [3]. Yue et al. (2016) forecasted the new energy vehicle ownership by the multiple linear regression method [4]. Apart from using mathematical methods to calculate the ownership, scholars are trying to figure out what makes vehicles attract customers. Barth et al. (2016) explored the current perspective of potential EV users from Germany on electric mobility and to identify predictors of a general goal intention to use EVs [5]. Prateek and Kara (2017) proposed a new simulation-based fleet evolution framework to forecast Americans' long-term adoption levels of connected and autonomous vehicle technologies under eight different scenarios [6]. Bass diffusion model has been widely used in new product promotion, including the EV. Ming et al. (2013) used the Bass model to predict the number of EV and analyzed the amount of EV in China [7]. Bin et al. (2013) proposed an innovation diffusion model for Chinese electric vehicles (EVs) based on the extension of generalized Bass model considering infrastructure and price reduction effects [8]. Yingqi et al. (2016) took Toyota Prius' international data for reference and combined it with the characteristics of the Chinese market to build the Bass prediction model of China's new energy vehicles market sales and then used the 
model to predict the trend of the whole new energy vehicles industry, pure electric vehicles, and plug-in hybrid vehicles industry [9]. In view of the limited historical data, in the estimation of the parameters of the Bass model of the electric vehicle in China, the parameters of BEV in China were determined by comparing the parameters of other consumer products. In summary, some achievements have been made in the application of Bass model in the prediction of new energy automotive industry.

Compared with Bass model, LV model has been used for predicting market share, transition of silicon wafers, technology substitution, and market competition, which seems to be a natural way of portraying the competitive struggle in a market [10-15]. H.-T. Wang and T.-C. Wang (2016) used LV model to study on the diffusion and competition of TV and smart-phone industries [16]. Haijun et al. (2011) proposed a method for estimation the parameters of Lotka-Volterra model based on the grey direct modeling [17]. Wu et al. (2012) analyzed the long-term relationship between the two variables to predict the values of two variables in the social system or economic system [18]. In the present paper, Lotka-Volterra model has been used to predict the ownership of CVs and BEVs. Lotka-Volterra model is a classical method to simulate natural ecosystems, especially when it is used for population ecosystem. This model and its mathematical expressions are widely applied for describing different populations competing for environment resources and the relations among them. There are three classical relations of Lotka-Volterra model. They are competitive relation, predator-prey relation, and symbiotic relation. The model can be used for predicting certain system's change in the future and speculate certain populations' growth or extinction. What is more, this model can explain how the system changes. The relations among these three type vehicles are unknown. The real relations need to be simulated and then verified. Lotka-Volterra model focuses on the relation between homogeneous species. LV model has been used to forecast the development of other industry, such as TV and mobile phone. We try to explore the forecasting accuracy between LV models initially based on the benchmark of Bass model.

\section{Methodology}

2.1. Bass Model. Bass model is a model for the prediction of the market share of the innovation products, technology adoption, and diffusion. The core assumption of Bass model is that the adoption of innovator is independent of other members of the social system. However, the time for the adoption of the new product is influenced by the pressure of the social system, and the pressure increases with the increase of the number of people who use it earlier. The potential users are called imitator. For example, Massiania and Gohsb (2015) investigated the potential for the use of the Bass diffusion model to promote the market diffusion of electric vehicles in Germany [19].

The expression of Bass diffusion model is

$$
n(t)=\frac{d N(t)}{d t}=p[m-N(t)]+\frac{q}{m} N(t)[m-N(t)] .
$$

The meaning of the mathematical expression and parameter of (1) is explained as follows:

(1) $d N(t) / d t$ is the number of noncumulative adopters at $t$.

(2) $N(t)$ is the number of cumulative adopters at $t$.

(3) $m$ is the biggest marker potential.

(4) $p$ is the external influence coefficient (also called innovation coefficient).

(5) $q$ is the internal influence coefficient (also called imitation coefficient).

(6) $p[m-N(t)]$ represents the number of adopters whose purchase is influenced by external factors, which are called innovation adopters because they are not influenced by people who have already used this product.

(7) $q / m * N(t) *[m-N(t)]$ represents the number of adopters whose purchase is influenced by internal factors, which are called imitators because they are influenced by people who have already used this product.

The Bass model expresses the essence of the diffusion process with mathematical equations, which greatly simplifies the understanding of the diffusion of innovation. The basic Bass model is based on a series of important assumptions. Nonetheless, the basic Bass model does not consider the impact of marketing strategy on the diffusion of innovation products. In view of these defects, Bass added decision variables into the Bass model and proposed a generalized Bass model.

$$
\begin{aligned}
n(t) & =\frac{d N(t)}{d t} \\
& =\left\{p[m-N(t)]+\frac{q}{m} N(t)[m-N(t)]\right\} * x(t) .
\end{aligned}
$$

In (2), $x(t)$ is a modified variable which depends on decision variables.

$$
x(t)=1+\left[\frac{\Delta \operatorname{Pr}(t)}{\operatorname{Pr}(t-1)}\right] \beta_{1}+\left[\frac{\Delta \operatorname{ADV}(t)}{\operatorname{ADV}(t-1)}\right] \beta_{2} .
$$

In (3), $\operatorname{Pr}(t)$ represents the innovation product price on $t$; $\operatorname{ADV}(t)$ represents the advertisement cost for this product; $\beta_{1}$ and $\beta_{2}$ are two coefficients which represent the price impact and advertisement cost impact, respectively. When $x(t)$ is a constant, the generalized Bass model is equivalent to the original Bass model.

2.2. Lotka-Volterra Model. Lotka-Volterra model is a classical method to simulate natural ecosystems, especially when it is used for population ecosystem. The model and its mathematical expressions are widely applied for describing different populations competing for environment resources and the relations among them. The model can be used for 
predicting certain system's change in the future and speculate certain populations' growth or extinction.

$$
\begin{aligned}
& \frac{d X(t)}{d t}=r_{1} * X(t)\left(1-\frac{a_{11} X}{k_{1}}-\frac{a_{21} Y}{k_{2}}\right) \\
& \frac{d Y(t)}{d t}=r_{2} * Y(t)\left(1-\frac{a_{12} X}{k_{1}}-\frac{a_{22} Y}{k_{2}}\right) .
\end{aligned}
$$

In (4), $X(t)$ represents the amount of population 1 at $t$ time; $Y(t)$ represents the amount of population 2 at $t$ time; $r_{i}$ represents the growth ratio of $i$ population; $a_{i j}$ represents the competition between populations $i$ and $j$; $k_{i}$ represents the maximum number of species that can be carried by the environment.

\section{Data and Result}

3.1. Simulation of BEV Based on Bass Model. According to the analysis of the current situation of BEV in China, it is necessary to modify the original market efficiency function $x(t)$ of basic Bass model. First of all, automotive consumer's purchase decision is affected by the price, which will be affected by the cost of the future operation, so the fluctuation of fuel prices should also be taken into account. Secondly, due to the fact that the BEV is still in the market introduction stage, low battery capacity results in shorter mileage. Meanwhile, the construction of infrastructure such as charging station and charging pile is more important than the promotion of advertising. Therefore, the present paper will use the number of charging facilities to replace the impact of advertising on the spread of BEV

$$
\begin{aligned}
x(t)= & +\left[\frac{\Delta \operatorname{Pv}(t)}{\operatorname{Pv}(t-1)}-\frac{\Delta \operatorname{Pg}(t)}{\operatorname{Pg}(t-1)}\right] \beta_{1} \\
& +\max \left\{0,\left[\frac{\Delta \operatorname{Cs}(t)}{\operatorname{Cs}(t-1)}\right]\right\} \beta_{2},
\end{aligned}
$$

where $\operatorname{Pv}(t)$ is the ratio of electric vehicle price to traditional vehicle price; $\operatorname{Pg}(t)$ is the gas price on $t$; $\operatorname{Cs}(t)$ is the amount of new charging stations. $\beta_{1}$ is the price impact coefficient, while $\beta_{2}$ is the infrastructure impact coefficient.

As a new innovative product, $\mathrm{BEV}$, its market potential depends on the maturity of the technology of the product, the coverage of public facilities, and the amount of government subsidies. The amount of BEV ownership from Jan 2015 to Sep 2016 in China is shown in Table 1. The statistics data of BEV is from National Bureau of Statistics of China (http://www.stats.gov.cn/).

Despite the fact that, currently, national and local governments are promoting the development of BEV in China, but the effectiveness of BEV is still not as expected by most consumers. In 2012, the State Council of China passed the "energy saving and new energy automotive industry development plan (2012-2020)," which mentioned that, in 2015, the battery EV and plug-in hybrid EV production and sales volume would reach 500 thousand units and in 2020 the cumulative production and sales would reach more than 5 million vehicles. Based on the plan, the maximum market
TABLE 1: The amount of BEV ownership from Jan 2015 to Sep 2016 in China.

\begin{tabular}{lc}
\hline BEV & Month \\
\hline 273927 & Jan 2015 \\
279972 & Feb 2015 \\
294094 & Mar 2015 \\
302414 & Apr 2015 \\
313270 & May 2015 \\
340224 & Jun 2015 \\
357062 & Jul 2015 \\
375962 & Aug 2015 \\
404054 & Sep 2015 \\
438370 & Oct 2015 \\
463034 & Nov 2015 \\
583200 & Dec 2015 \\
604926 & Jan 2016 \\
618926 & Feb 2016 \\
641862 & Mar 2016 \\
673634 & Apr 2016 \\
708634 & May 2016 \\
752634 & Jun 2016 \\
788634 & Jul 2016 \\
826634 & Aug 2016 \\
870634 & Sep 2016 \\
\hline
\end{tabular}

potential is assumed to be 5 million units. Through iterative calculation, $p$ and $q$ in (2) are fitted.

$$
\begin{aligned}
n(t)= & \frac{d N(t)}{d t} \\
= & -0.0013 *[5000000-N(t)] \\
& +\frac{0.0839}{5000000} N(t)[5000000-N(t)] .
\end{aligned}
$$

After the model is confirmed, Matlab is used for simulating the fitting model to get the forecast result of $\mathrm{BEV}$, which is shown in Table 2.

3.2. Simulation of BEV Based on LV Model. BEV and conventional car $(\mathrm{CV})$ are the two most popular products in the current automotive market. Therefore, only these two kinds are discussed for forecast the future vehicle ownership in China. The LV model can be modified similar to the Bass diffusion model; the parameter $K$ represents the maximum number of species that can be carried by the environment. For CV, the number of Chinese civil car ownerships has exceeded 200 million. According to the average per capita car ownership, the largest market capacity can be 400 million. The unknown parameters involved in (4) are more than 10, and two $K$ values are calibrated with the following 8 unknown parameters. $r_{i}$ can be estimated by the average growth rate method. Based on the observed data of BEV and CV, $r_{1}$ is 
TABLE 2: Simulated result of BEV based on Bass model.

\begin{tabular}{lcc}
\hline BEV (simulated data) & BEV (observed data) & Month \\
\hline 266568 & 273927 & Jan 2015 \\
282173 & 279972 & Feb 2015 \\
299006 & 294094 & Mar 2015 \\
317153 & 302414 & Apr 2015 \\
336704 & 313270 & May 2015 \\
357755 & 340224 & Jun 2015 \\
380396 & 357062 & Jul 2015 \\
404730 & 375962 & Aug 2015 \\
430875 & 404054 & Sep 2015 \\
458946 & 438370 & Oct 2015 \\
489056 & 463034 & Nov 2015 \\
521320 & 583200 & Dec 2015 \\
555849 & 604926 & Jan 2016 \\
592753 & 618926 & Feb 2016 \\
632143 & 641862 & Mar 2016 \\
674127 & 673634 & Apr 2016 \\
718812 & 708634 & May 2016 \\
766304 & 752634 & Jun 2016 \\
816705 & 788634 & Jul 2016 \\
870060 & 826634 & Aug 2016 \\
926463 & 870634 & Sep 2016 \\
\hline
\end{tabular}

calculated as 0.0108 and $r_{2}$ is calculated as 0.06054 . Then the equation set can be fitted as shown in

$$
\begin{aligned}
& \frac{d X(t)}{d t}=0.01087 * X(t)\left(1-\frac{0.7288 X}{4 * 10^{8}}+\frac{3.156 Y}{5 * 10^{6}}\right) \\
& \frac{d Y(t)}{d t}=0.06054 * Y(t)\left(1+\frac{11.20 X}{4 * 10^{8}}-\frac{2.445 Y}{5 * 10^{6}}\right) .
\end{aligned}
$$

Matlab is used for simulating the fitting model and two data groups are listed. The observed data and simulated of BEV and CV are shown in Table 3.

3.3. Simulation Accuracy Comparison of the Two Models. The mean absolute percentage error (MAPE) as shown in (8) is designed for evaluating the forecast accuracy. The higher the MAPE is, the less accurate the model is:

$$
\text { MAPE }=\frac{1}{n} \sum_{i=1}^{n}\left|\frac{a_{i}-a_{i}^{*}}{a_{i}}\right| * 100 \% .
$$

In (8), $a_{i}$ is the actual observed value; $a_{i}^{*}$ is the simulated value. Prediction accuracy level divided by MAPE value is shown in Table 4.

As a kind of new technology products, electric vehicle has entered into the mature automobile market. Bass model is widely used in the market diffusion of a single new product, while LV model takes no less than two species into account and it is used for forecasting less frequently. In order to compare the accuracy of these two models, MAPE is used for calculating forecasting errors.

As Table 5 shows, the two models have good prediction accuracy, but the Bass model is more accurate. Part of the

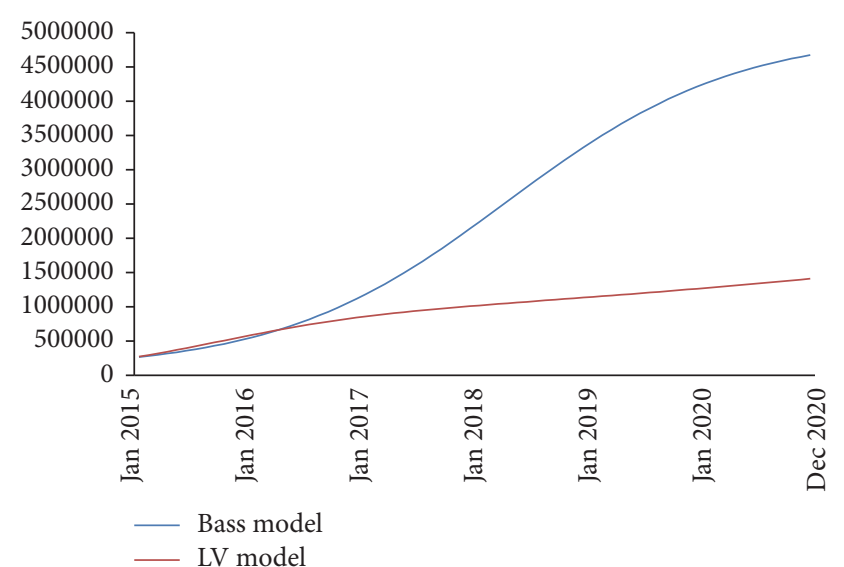

FIGURE 1: Forecasting of BEV ownership from 2017 to 2020 based on the two models.

reason is that the LV model is more complex than the Bass model, so the variance is greater with the iterative fitting of the unknown parameters, resulting in higher MAPE value. In addition, Bass model has only one dimension and focuses on the diffusion trend while LV model has two dimensions and mainly describes the relationship and competing process between the two populations. According to the MAPE value, Bass model is proved to have better simulation capacity because of the model's precise expression.

3.4. Forecasting of BEV Ownership from 2017 to 2020. After comparison of simulation accuracy comparison, the present paper gives the forecast of BEV ownership from 2017 to 2020 as shown in Table 6 and Figure 1. From Jan 2015 to Sept 2016, the simulation results are close between the two models, while the forecasting results are greatly different. The forecasting value of Bass model keeps growing rapidly while that of $\mathrm{LV}$ model increases slowly.

The Bass model only considers the time series and does not take into account the factors that affect the development of new energy vehicles. Moreover, the forecasting result needs the sustainable development of the policy and the stability of the market environment as the guarantee condition. If the industrial policy and the market environment fluctuate greatly, the forecasting result is no longer applicable. The maximum market volume is assumed to be 5 million. For the Bass model, it will be reached in 2022 while it will only reach 1.58 million for LV model in 2022.

\section{Conclusion}

To compare the prediction accuracy, the present paper fitted the parameters of modified Bass and the LV model. The two models have good prediction accuracy, but the Bass model is more accurate. Part of the reason is that the LV model is more complex than the Bass model, so the variance is greater when the iterative fitting of the unknown parameters, resulting in higher MAPE value. In addition, Bass model has only one dimension and focuses on the diffusion trend while LV model has two dimensions and mainly describes 
TABLE 3: Simulated result of BEV and CV based on LV model.

\begin{tabular}{|c|c|c|c|c|}
\hline $\begin{array}{l}\mathrm{BEV} \\
\text { (simulated data) }\end{array}$ & $\begin{array}{c}\text { BEV } \\
\text { (observed data) }\end{array}$ & $\begin{array}{c}\text { CV } \\
\text { (simulated data) }\end{array}$ & $\begin{array}{c}\mathrm{CV} \\
\text { (observed data) }\end{array}$ & Month \\
\hline 266567 & 273927 & 155969992 & 156244073 & Jan 2015 \\
\hline 282173 & 279972 & 157493647 & 157634728 & Feb 2015 \\
\hline 299006 & 294094 & 159052768 & 159491006 & Mar 2015 \\
\hline 317153 & 302414 & 160649120 & 161151486 & Apr 2015 \\
\hline 336704 & 313270 & 162284410 & 162749930 & May 2015 \\
\hline 357755 & 340224 & 163960281 & 164234376 & Jun 2015 \\
\hline 380396 & 357062 & 165678480 & 165486138 & Jul 2015 \\
\hline 404730 & 375962 & 167440505 & 166885738 & Aug 2015 \\
\hline 430875 & 404054 & 169247482 & 168608846 & Sep 2015 \\
\hline 458946 & 438370 & 171100413 & 170511430 & Oct 2015 \\
\hline 489056 & 463034 & 173000174 & 172683566 & Nov 2015 \\
\hline 521320 & 583200 & 174947514 & 175005500 & Dec 2015 \\
\hline 555849 & 604926 & 176943055 & 177373774 & Jan 2016 \\
\hline 592753 & 618926 & 178987296 & 179056774 & Feb 2016 \\
\hline 632143 & 641862 & 181080607 & 181473838 & Mar 2016 \\
\hline 674127 & 673634 & 183223234 & 183564066 & Apr 2016 \\
\hline 718812 & 708634 & 185415293 & 185621066 & May 2016 \\
\hline 766304 & 752634 & 187656780 & 187648066 & Jun 2016 \\
\hline 816705 & 788634 & 189947559 & 189464066 & Jul 2016 \\
\hline 870060 & 826634 & 192287371 & 191497066 & Aug 2016 \\
\hline 926463 & 870634 & 194675689 & 194017066 & Sep 2016 \\
\hline
\end{tabular}

TABle 4: Prediction accuracy level divided by MAPE value.

\begin{tabular}{lc}
\hline MAPE\% & Prediction capability \\
\hline$<10$ & Highly accurate \\
$10-20$ & Good \\
$20-50$ & Reasonable \\
$>50$ & Inaccurate \\
\hline
\end{tabular}

TABLE 5: MAPE Comparison between LV and Bass model.

\begin{tabular}{lc}
\hline & MAPE \\
\hline Bass & $4.6 \%$ \\
LV & $9.1 \%$ \\
\hline
\end{tabular}

TABLE 6: Forecasting of BEV ownership from 2017 to 2020 based on the two models.

\begin{tabular}{lcccc}
\hline & 2017 & 2018 & 2019 & 2020 \\
\hline Bass model & 2138387 & 3325383 & 4209993 & 4674325 \\
LV model & 1008591 & 1134000 & 1263081 & 1410821 \\
\hline
\end{tabular}

the relationship and competing process between the two populations. According to the MAPE value, Bass model is proved to have better simulation capacity because of the model's precise expression.

There are still some differences between the results of the prediction and the observed formulation. On the one hand, the new energy vehicles in the Chinese market are still in the policy support phase. The growth of new energy vehicles depends largely on the amount of subsidies and the corresponding tax benefits policy. The correspondent facilities cannot dispel consumer concerns. Therefore, the existing data based on time series has strong policy relevance. On the other hand, to some extent, the new energy vehicles have technical defects. Therefore, the forecasting methods cannot adapt to the new energy vehicles after the technical improvement. After a comparative study, it is found that although the Bass model is not able to consider the external factors, but its prediction accuracy is better than the LV model.

\section{Conflicts of Interest}

The authors declare that they have no conflicts of interest.

\section{Acknowledgments}

This study is supported by the National Natural Science Foundation of China (51508432), the Natural Science Foundation of Hubei (2014CFB850), and the Fundamental Research Funds for the Central Universities of China (2014-IV-034).

\section{References}

[1] W. Yini, "Car ownership forecast in China-an analysis based on Gompertz equation," Research on Financial and Economic Issues, vol. 11, pp. 44-50, 2005. 
[2] J. Gu, F. Qi, and J. Wu, "Forecasting on China's civil automobileowned based on Gompertz model," Technology Economics, vol. 29, no. 1, pp. 57-62, 2010.

[3] J. Ma, N. Wang, and D. Kong, "Market forecasting modeling study for new energy vehicle based on AHP and logit regression," Journal of Tongji University, vol. 37, no. 8, pp. 1079-1084, 2009.

[4] Z. Yue, D. Zhang, K. Cai et al., "Research on the development trend of new energy vehicle market," Science and Technology Economic Guide, vol. 33, pp. 47-49, 2016.

[5] M. Barth, P. Jugert, and I. Fritsche, "Still underdetected - social norms and collective efficacy predict the acceptance of electric vehicles in Germany," Transportation Research Part F: Traffic Psychology and Behaviour, vol. 37, pp. 64-77, 2016.

[6] B. Prateek and M. K. Kara, "Forecasting Americans long-term adoption of connected and autonomous vehicle technologies," Transportation Research Part A, vol. 95, pp. 49-63, 2017.

[7] Z. Ming, Z. Fanxiao, Z. Xiaoli, and X. Song, "Forecast of electric vehicles in China based on bass model," Electric Power, vol. 46, no. 1, pp. 37-39, 2013.

[8] R. Bin, S. Luning, and Y. Jianxin, "Development of a generalized bass model for Chinese electric vehicles based on innovation diffusion theory," Ruankexue, vol. 27, no. 4, pp. 17-22, 2013.

[9] L. Yingqi, W. Meng, and W. Jingyu, "The predictive research on China’s new energy vehicles market," CNKI Journal, vol. 37, no. 4, pp. 87-91, 2016.

[10] A. Marasco, A. Picucci, and A. Romano, "Market share dynamics using Lotka-Volterra models," Technological Forecasting and Social Change, vol. 105, pp. 49-62, 2016.

[11] T. Modis, "Insights on competition from a science-based analysis," Advances in Psychology Research, vol. 88, pp. 1-25, 2011.

[12] S.-Y. Chiang, "An application of Lotka-Volterra model to Taiwan's transition from $200 \mathrm{~mm}$ to $300 \mathrm{~mm}$ silicon wafers," Technological Forecasting and Social Change, vol. 79, no. 2, pp. 383-392, 2012.

[13] S. A. Morris and D. Pratt, "Analysis of the Lotka-Volterra competition equations as a technological substitution model," Technological Forecasting and Social Change, vol. 70, no. 2, pp. 103-133, 2003.

[14] V. B. Kreng and H. T. Wang, "The interaction of the market competition between LCD TV and PDP TV," Computers and Industrial Engineering, vol. 57, no. 4, pp. 1210-1217, 2009.

[15] H.-C. Hung, Y.-S. Tsai, and M.-C. Wu, "A modified LotkaVolterra model for competition forecasting in Taiwan's retail industry," Computers and Industrial Engineering, vol. 77, pp. 7079, 2014.

[16] H.-T. Wang and T.-C. Wang, "Application of the grey LotkaVolterra model to forecast the diffusion and competition analysis of the TV and smartphone industries," Technological Forecasting and Social Change, vol. 106, no. 5, pp. 37-44, 2016.

[17] C. Haijun, L. Xijuan, L. Jie et al., "Estimation the parameters of Lotka-Volterra model based on the grey direct modeling and its application," Mathematics in Practices and Theory, vol. 41, no. 23, pp. 108-113, 2011.

[18] L. Wu, S. Liu, and Y. Wang, "Grey Lotka-Volterra model and its application," Technological Forecasting and Social Change, vol. 79, no. 9, pp. 1720-1730, 2012.

[19] J. Massiania and A. Gohsb, "The choice of Bass model coefficients to forecast diffusion for innovative products: an empirical investigation for new automotive technologies," Research in Transportation Economics, vol. 50, pp. 17-28, 2015. 


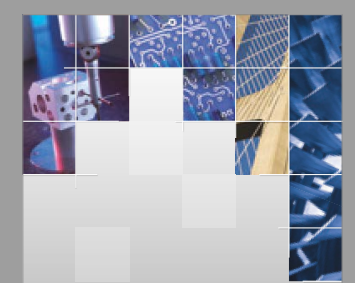

\section{Enfincering}
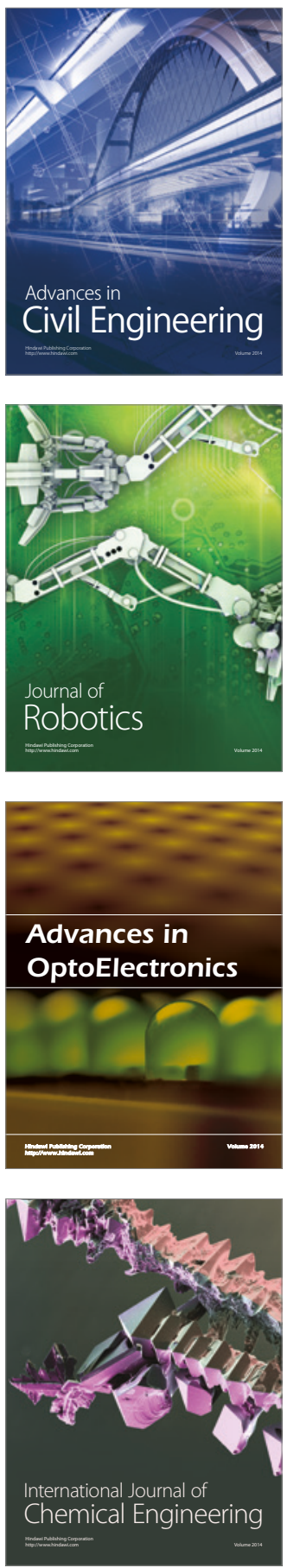

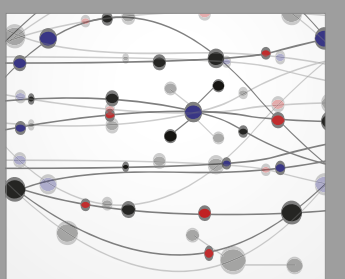

The Scientific World Journal

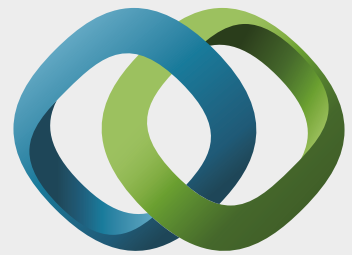

\section{Hindawi}

Submit your manuscripts at

https://www.hindawi.com
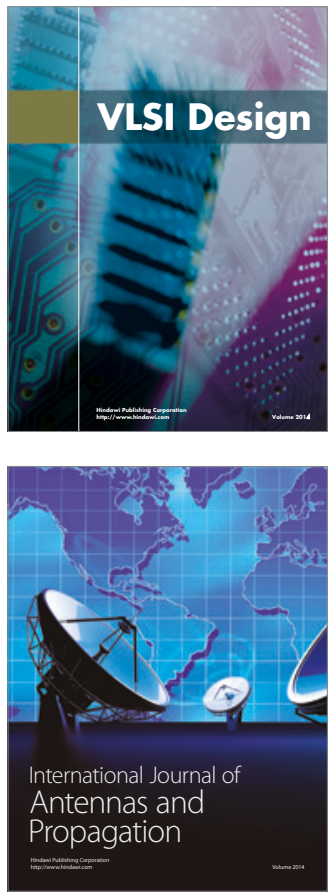

\section{Rotating}

Machinery
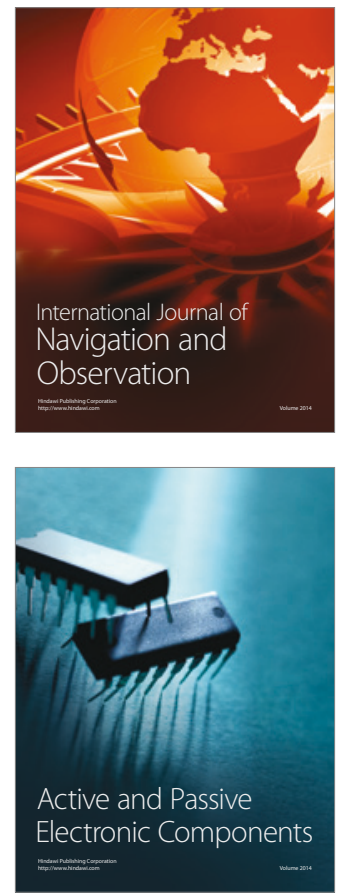
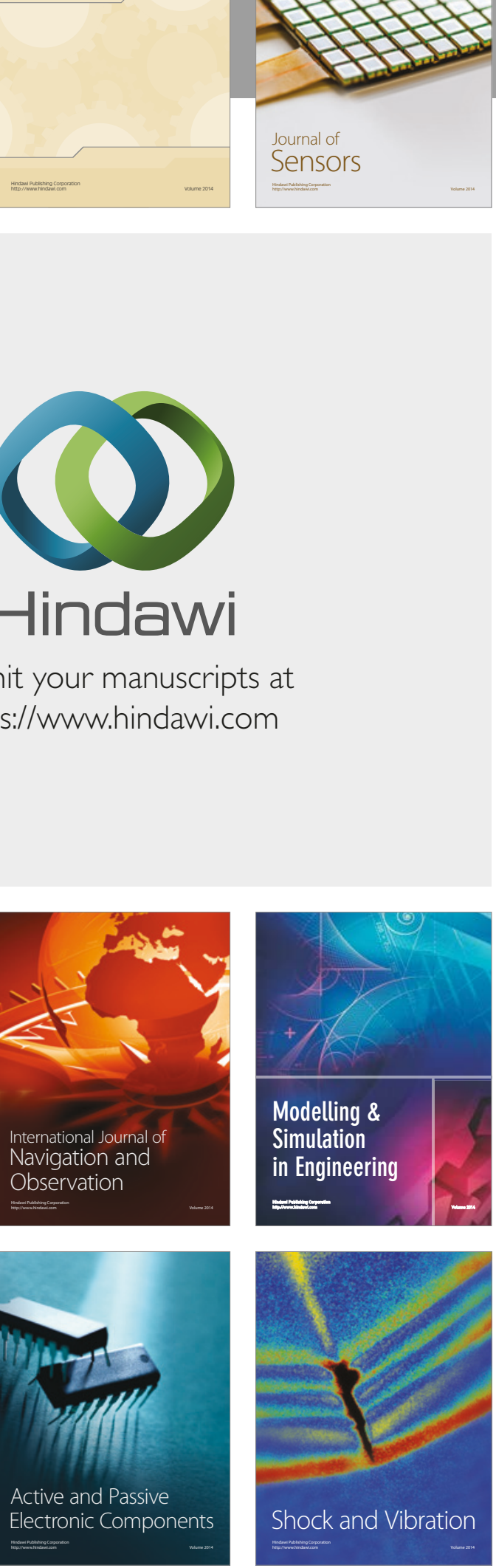
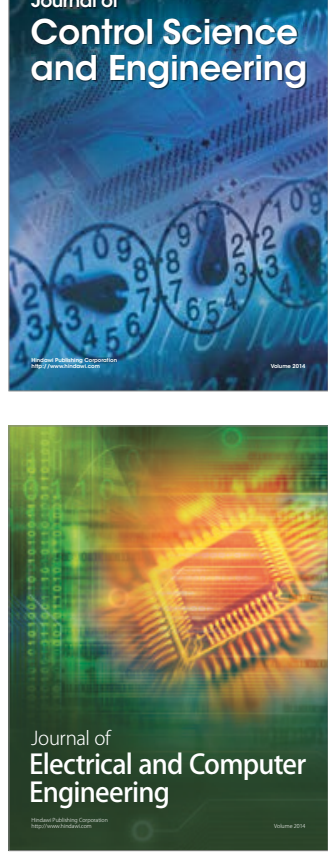

Distributed

Journal of

Control Science

and Engineering
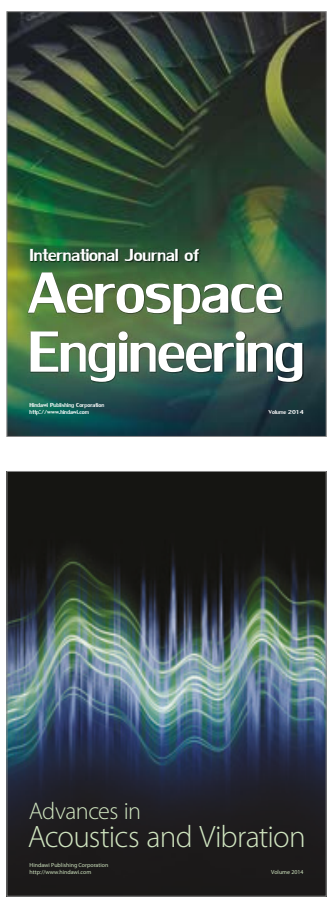

Sensor Networks 